\title{
Importance des caractéristiques personnelles et de l'environnement personnel dans le modèle entrepreneurial des petites entreprises
}

\author{
Yves Robichaud, Université Laurentienne ${ }^{1}$ \\ Egbert McGraw, Université de Moncton
}

\section{Introduction}

La majorité des modèles théoriques ${ }^{2}$ à l'étude de la performance entrepreneuriale fait ressortir, sans exception, les caractéristiques personnelles de l'entrepreneur et l'environnement personnel dans lequel il évolue comme deux des éléments clés de la réussite entrepreneuriale chez les petites entreprises ${ }^{3}$. L'attention manifestée par ces chercheurs à l'égard de ces deux variables se base sur les prémices que l'entrepreneur est au cœur des activités et des décisions de l'entreprise. Le sens plus prononcé d'identité de l'entrepreneur envers son entreprise suggère que les caractéristiques de l'entrepreneur ainsi que son environnement personnel peuvent exercer une influence importante sur la conduite de l'entreprise et sur sa performance.

Étant donné l'importance accordée à ces deux variables dans les modèles théoriques de la performance entrepreneuriale il devient, à notre avis, nécessaire d'approfondir les facteurs qui sont à l'origine de la présence des entrepreneurs en affaires ainsi que sur les effets du métier d'entrepreneur sur le lien travailfamille. Pour y parvenir, nous avons recours à des entretiens de recherche.

\section{Informations sur les variables de l'étude}

La définition des caractéristiques personnelles d'un entrepreneur retenue pour notre étude s'inspire, en partie, de la définition utilisée par Blawatt ${ }^{4}$, et comprend essentiellement les caractéristiques démographique, sociologique, familiale et psychologique de l'entrepreneur acquises ou développées durant son vécu.

D'abord les variables dites démographiques peuvent se résumer à l'âge, au niveau de scolarité, à l'expérience, aux antécédents familiaux, à l'état civil, au rang familial et au sexe. Bien que les variables démographiques exercent une influence certaine sur la décision d'un individu de démarrer sa propre entreprise, Greenberger et Sexton ${ }^{5}$ s'entendent sur le fait que ces variables ne permettent pas d'expliquer à elles seules le phénomène entrepreneurial.

Les caractéristiques familiales représentent les valeurs individuelles inculquées par la famille tout au long de la vie d'un individu. Selon Shapiro ${ }^{6}$, les parents, les enfants et la famille en générale créent un environnement qui peut influencer les intentions de comportement d'un individu. Il souligne que cette source est dominante dans la prédisposition d'un individu à devenir entrepreneur.

Quant aux caractéristiques psychologiques elles sont les traits de personnalité (attitudes et valeurs) généralement associés au fait d'être entrepreneur. Ces traits qui s'acquièrent aussi par le vécu (par exemple, l'éducation, l'expérience, les antécédents familiaux, etc.) déterminent les intentions de comportement des individus. Contrairement aux caractéristiques démographiques qui sont mesurées par des données recueillies directement, les caractéristiques psychologiques doivent être mesurées à l'aide d'un instrument de mesure validé. Les spécialistes ${ }^{7}$ s'entendent sur la pertinence 
de certains traits de personnalité dans le phénomène entrepreneurial. Parmi ceux les plus souvent cités, on relève les regroupements suivants : créativité et innovation, besoin d'indépendance et d'autonomie, besoin d'accomplissement, tendance à la prise de risques modérés et contrôle du destin. Cependant, selon Blawatt ${ }^{8}$ l'ensemble des recherches sur les déterminants psychologiques n'ont pas permis, à ce jour, d'établir de liens entre les traits de personnalité et la réussite en affaires, ni d'identifier les traits qui permettent de différencier les entrepreneurs des non entrepreneurs.

Selon la plupart des sociologues, la connaissance des facteurs historiques que nous venons de présenter est importante puisqu'ils sont à l'origine du système de croyances et de valeurs développé par chaque individu durant son vécu. C'est entre autres ce système de croyances et de valeurs qui déterminerait le degré d'intention ou de prédisposition d'un individu de se lancer en affaires.

Par ailleurs, les recherches portant sur l'environnement personnel de l'entrepreneur, et plus spécifiquement celles effectuées sur le lien travail-famille, sont associées étroitement aux recherches menées sur les femmes en affaires ${ }^{9}$. Comme la plupart des entrepreneurs masculins, les femmes en affaires sont mariées et ont des enfants. Cependant contrairement aux hommes, ce sont elles qui doivent supporter les responsabilités liées au travail de la maison. À ce sujet, l'étude de Newcomer et Taylor ${ }^{10}$ rapporte ce qui suit : «The Industrial Revolution changed the nature of the family and moved away from a homed-based family work unit in which husband and wife participated fully both in the raising and instruction of children. With means of production concentrated in factories and a 12-hourworkday, the norm for most employees, men became the breadwinners of the family unit [...] In spite of the technological revolution that improved productivity in the business world, the American housewife consistently put in approximately 50 hours a week on domestic chores from 1920 to 1990 [...] Our culture still expects women to assume the primary responsability for home and family obligations » (2003). Ce point de vue est également partagé par Carter ${ }^{11}$ qui rapporte que les hommes ne supportent pas suffisamment leur conjointe au niveau des responsabilités familiales.

D'autres recherches ${ }^{12}$ ont trouvé que la gestion des conflits entre les responsabilités personnelles et celles liées au travail était le problème le plus souvent mentionné par les femmes entrepreneures. Ainsi, d'autres études ${ }^{13}$ ont identifié un certain nombre de facteurs qui sont à l'origine de ces conflits, notamment un manque de support de la famille, un revenu instable et incertain, le risque de pertes financières, de longues heures de travail ainsi qu'un niveau élevé de stress. L'équilibre entre les responsabilités familiales et les exigences de l'entreprise est une source de pression pour les femmes en affaires. Cette source de pression se traduit par le manque de temps et d'énergie pour faire ce qu'il y a à faire (p. ex., réfléchir aux problèmes financiers et fixer des priorités), par un manque de vie sociale et par des pressions de leur famille pour être plus disponible. Plusieurs chercheurs ${ }^{14}$ concluent que les entrepreneurs doivent réaliser que le démarrage d'une entreprise et les responsabilités familiales ne s'harmonisent pas tellement bien et ajoutent que la famille et l'entreprise constituent un système intégré et que l'éclatement d'un de ces deux éléments affectera l'autre.

\section{Les entrepreneurs doivent réaliser que le démarrage d'une entreprise et les responsabilités familiales ne s'harmonisent pas tellement bien.}

\section{Méthodologie}

\section{Échantillon et critères de sélection}

Notre approche est fondée sur des entretiens de recherche. L'entretien qualitatif est l'approche privilégiée dans le cadre de notre recherche. Plus particulièrement, nous avons retenu l'entretien centré, guidé ou à réponses libres.

Notre échantillon compte vingt-huit entreprises des secteurs de service et de détail de deux régions du Nouveau-Brunswick. Les entreprises constituant l'échantillon ont été sélectionnées de façon non probabiliste. Seules les entreprises de services et de détail ont été retenues, qu'elles aient été créées par les entrepreneurs ou qu'elles soient issues de rachats, de legs ou de franchises. Pour être retenus, les propriétairesdirigeants de ces entreprises devaient être propriétaires majoritaires (c'est-à-dire posséder l'entreprise dans une proportion de $50 \%$ et plus) et satisfaire à notre définition de l'entrepreneur, à savoir « un individu qui con- 
tribue au capital de l'entreprise et qui participe aux activités quotidiennes de celle-ci ».

\section{Procédure d'expérimentation}

Des appels téléphoniques ont d'abord été effectués pour trouver des entrepreneurs répondant aux critères d'échantillonnage décrits précédemment et qui accepteraient de participer à l'étude. À cet effet, les participants éventuels ont été sélectionnés à partir des répertoires des commissions industrielles des régions concernées. Toutes les entreprises contactées ont accepté de participer à l'étude. Certaines entreprises auxquelles on a demandé de participer étaient connues des chercheurs.

Le déroulement typique d'un entretien peut se résumer de la façon suivante: après une période d'échanges informels, on rappelait brièvement au participant les objectifs de la recherche et de l'entretien. On repassait alors les thèmes couverts par l'entretien en signalant qu'il ne durerait pas plus de trente minutes. Avec l'approbation des répondants, les entretiens furent enregistrés pour s'assurer une transcription plus fidèle.

La transcription du matériel s'est effectuée autant que possible dans une attitude impartiale, ouverte, en évitant les a priori. Pour fins d'analyse des données, nous avons créé une grille d'analyse ou une fiche synthèse qui a été utilisée dans le cadre de chaque analyse. Sur cette fiche, nous avons consigné des renseignements d'ordre général (y compris le nom de l'interviewé, le nombre d'employés et le comté) et les passages des entretiens jugés importants pour chacun des thèmes couverts. Ces passages ou ces extraits ont ensuite été retranscrits sur la grille d'analyse, puis nous en avons fait l'analyse pour faire ressortir les fréquences d'apparition, organiser les catégories et établir des liens ou des relations. Les résultats de cette analyse sont présentés dans la section qui suit.

\section{Résultats}

\section{Profil des répondants}

Le tableau 1 résume certains des renseignements recueillis auprès des répondants $(5$ des 28 répondants n'ont pas rempli le questionnaire complémentaire donné à la fin de l'entretien). Les résultats obtenus révèlent une répartition assez bonne des répondants aux plans $d u$ sexe, du chiffre d'affaires, de l'âge de l'entreprise, de l'expérience et du niveau de scolarité, contrairement aux antécédents en affaires et les débuts dans l'entreprise où l'on remarque que les répondants ont favorisé la création de leur entreprise par rapport à l'acquisition, et une moins grande expérience dans les affaires.

\section{Tableau 1}

Profil des répondants $(\mathrm{n}=\mathbf{2 3})$

\begin{tabular}{lccc}
\multicolumn{1}{c}{ Variables } & Hommes & Femmes & Total \\
Sexe & 12 & 11 & 23 \\
Aîné de la famille & 5 & 2 & 7 \\
- Oui & 7 & 9 & 16 \\
- Non & & & \\
Statut marital & & & \\
- Marié/union libre & 8 & 2 & 6 \\
- Célibataire & 4 & & \\
Nombre d'enfants & & 2 & 4 \\
- Aucun & 2 & 2 & 3 \\
- 1 enfant & 1 & 4 & 5 \\
- 2 enfants & 7 & 3 & \\
- 3 enfants et + & 2 & & 4.90 \\
Expérience (moyenne) & & 4.30 & 5.54 \\
- Direction & & 6.00 &
\end{tabular}




\begin{tabular}{|c|c|c|c|}
\hline Variables & Hommes & Femmes & Total \\
\hline $\begin{array}{l}\text { Débuts dans l'entreprise } \\
\text { - Création } \\
\text { - Acquisition }\end{array}$ & $\begin{array}{l}7 \\
5\end{array}$ & $\begin{array}{l}8 \\
3\end{array}$ & $\begin{array}{c}15 \\
8\end{array}$ \\
\hline $\begin{array}{l}\text { Âge de l'entreprise } \\
\text { - } 5 \text { ans et - } \\
\text { - } 6 \text { ans - } 10 \text { ans } \\
\text { - } 11 \text { ans }-20 \text { ans }\end{array}$ & $\begin{array}{l}4 \\
2 \\
6\end{array}$ & $\begin{array}{l}3 \\
3 \\
5\end{array}$ & $\begin{array}{c}7 \\
5 \\
11\end{array}$ \\
\hline $\begin{array}{l}\text { Antécédents en affaires } \\
\text { - Oui (déjà été en affaires) } \\
\text { - Non (jamais été en affaires) }\end{array}$ & $\begin{array}{l}3 \\
9\end{array}$ & $\begin{array}{l}4 \\
7\end{array}$ & $\begin{array}{c}7 \\
16\end{array}$ \\
\hline $\begin{array}{l}\text { Scolarité } \\
\text { - Secondaire et moins } \\
\text { - Collégial } \\
\text { - Universitaire }\end{array}$ & $\begin{array}{l}7 \\
4 \\
3\end{array}$ & $\begin{array}{l}4 \\
2 \\
3\end{array}$ & $\begin{array}{c}11 \\
6 \\
6\end{array}$ \\
\hline Âge de l'entrepreneur (moyenne) & 39.25 & 40.91 & 40.04 \\
\hline $\begin{array}{l}\text { Chiffre d'affaires } \\
\cdot 0-100000 \$ \\
\text { - } 100001 \$-500000 \$ \\
\text { - } 500001 \$ \text { et plus }\end{array}$ & $\begin{array}{l}1 \\
8 \\
3\end{array}$ & $\begin{array}{l}4 \\
4 \\
3\end{array}$ & $\begin{array}{c}5 \\
12 \\
6\end{array}$ \\
\hline $\begin{array}{l}\text { Nombre d'employés } \\
\text { - } 5 \text { employés et - } \\
\text { - } 6 \text { employés }-10 \text { employés } \\
\text { - } 11 \text { employés et }+\end{array}$ & $\begin{array}{l}6 \\
2 \\
4\end{array}$ & $\begin{array}{l}7 \\
1 \\
3\end{array}$ & $\begin{array}{l}13 \\
3 \\
7\end{array}$ \\
\hline
\end{tabular}

\section{Facteurs historiques ayant contribué au choix d'une carrière en entrepreneurship}

L'analyse des entretiens fait ressortir trois grands facteurs qui ont prédisposé les répondants à choisir une carrière en affaires. Le premier facteur est le fait que l'un ou les deux parents des répondants sont ou ont été en affaires (18 des 28 répondants). Bien que ces répondants n'aient pas indiqué que la présence de leurs parents dans le milieu des affaires les avait directement motivés à démarrer leur propre entreprise, certains d'entre eux ont mentionné que leurs parents étaient pour quelque chose dans leur choix de carrière. Voici des extraits de commentaires à ce sujet :

Mon mari a commencé sa propre entreprise. Dans sa famille, il y en a plusieurs qui sont en affaires. Dans ma famille, j'ai aussi des sœurs qui sont en affaires. Mais pour vous dire que c'est ça qui m'a motivée, je ne le sais pas. (J.V.)

Mon père a toujours eu une entreprise dans le soussol de notre maison. Je ne sais pas si c'est ça ou pas qui m'a influencée. Aujourd'hui, il a une autre entreprise ici, en ville. Mais je n'ai jamais eu l'idée toute jeune de partir une entreprise. Peut-être que c'est quelque chose qui se prépare inconsciemment, je ne sais pas. La preuve, tu ne fais pas une maîtrise en administration des affaires pour ouvrir ta propre entreprise. (J.L.)

Mon père a été propriétaire de plusieurs entreprises. $C$ 'est le type de gars qui a toujours voulu être en affaires. J'ai donc vécu là-dedans, et même avant d'entrer à l'université, on discutait souvent de la possibilité d'aller en affaires. Il y a aussi un oncle de qui je suis proche et qui a toujours été en affaires. On jasait souvent de ça aussi. Peut-être que je ne serais pas en affaires maintenant si je n'avais pas eu ces influences-là. (D.R.)

Mes parents possédaient une entreprise, et ma mère m'a toujours dit qu'elle serait là pour m'aider si j'ouvrais mon commerce. Elle a 75 ans maintenant et elle m'aide encore aujourd'hui. (C.R.)

Mon père a toujours été en affaires, c'était un homme d'affaires. C'est lui qui m'a motivé à aller en affaires. Il me disait tout le temps : "Si tu veux avoir quelque chose à toi, il faut que tu fonces. » (B.C.)

Le deuxième facteur, qui concerne ceux et celles dont aucun des parents n'était en affaires, est la présence de 
parents très dynamiques, en particulier la mère. Les répondants de cette catégorie (6 des 28 répondants) ont dit qu'on leur avait appris très jeunes à se débrouiller et à devenir responsables. Certains de leurs commentaires sont reproduits ci-dessous :

On a été élevés jeunes à avoir beaucoup d'initiative. Ma mère a fait beaucoup d'efforts pour qu'on apprenne à se débrouiller et à être indépendants. À la maison, on pouvait aussi bien planter des clous que laver la vaisselle. (H.L.)

Je suis né dans une famille de 12, mais la source, c'était maman. Elle est venue au monde 50 ans avant son temps. Ma mère était une grande femme d'affaires même si elle n'a jamais été en affaires. À 45 ans, c'est elle qui est devenue la première personne à prendre la direction du syndicat à la coopérative [...] Elle était leader de nature, et c'est de là que ça vient. (R.T.)

Je ne sais pas si on peut parler d'hérédité, mais je crois que ça vient du côté de ma mère. Ma mère était fonceuse. Elle n'a jamais été en affaires parce que ce n'était pas tout le monde qui partait en " business " à 80 ans passés. Elle n'a jamais travaillé à l'extérieur, elle n'avait pas le temps, car on était 15 enfants. Moi, je vois chez ma mère des qualités de leader, mais je ne sais pas si c'est ça qui m'a amené à avoir une entreprise. (B.F.)

La dernière catégorie de répondants est composée des entrepreneurs n'ayant aucun antécédent en affaires et n'ayant pas non plus mentionné l'existence de compétences particulières dans leur famille (4 des 28 répondants). Ces individus ont dit avoir tout appris par euxmêmes. Ils ont acquis, au cours de leur vie, une grande expérience dans leur domaine d'activité, ce qui les a incités à démarrer leur propre entreprise. Voici ce que rapportent certains d'entre eux à ce sujet :

Personne de ma famille n'était en affaires. Je pense que c'est le fait que j'ai commencé très jeune à travailler. J'ai travaillé 12 ans dans un magasin d'alimentation et 18 ans dans une quincaillerie [...] J'ai toujours aimé cela. J'avais une certaine ambition dans ce que je faisais. À mon avis, c'est l'expérience de la vie qui m'a conduit jusqu'ici. (R.B.)

J'ai commencé très tôt, à l'âge de 13 ans, à travailler dans les restaurants. $\grave{A} 18$ ans, je travaillais à Montréal pour la propriétaire dont j'ai acheté le commerce par la suite. C'était dans le même domaine, soit des franchises de bar laitier. Huit ans après que j'ai ouvert le commerce [...] pour cette madame, elle m'a approchée pour me vendre le commerce. J'ai donc fini par acheter le commerce. Sans cette expérience-là, je ne pense pas que je serais ici aujourd'hui [...] Treize ans d'expérience dans le même domaine, ça ne me laissait pas grand choix [...] J'avais l'expérience et je savais où je m'en allais. (B.S.)

Personne dans nos familles n'était en affaires [...] Quand j'étais dans l'armée, je travaillais comme cuisinier, tout comme ma femme. Depuis que nous avons quitté l'armée, j'ai dû faire toutes sortes de jobs avant d'acheter ce commerce [...] C'est l'expérience de la vie, on a appris par nous-mêmes. (A.S.)

Enfin, parmi les autres facteurs qui ont été mentionnés et dont certains ressortent dans les extraits ci-dessus, on peut citer le fait d'avoir déjà été propriétaire d'une entreprise (trois répondants), d'avoir déjà travaillé dans sa jeunesse pour l'entreprise de ses parents (huit répondants), d'avoir travaillé pour l'entreprise avant de l'acquérir (quatre répondants) et d'avoir été marié à quelqu'un qui était en affaires avant d'ouvrir son propre commerce (deux répondants).

\section{L'appui apporté par le conjoint pouvait être d'ordre physique (implication directe dans le commerce ou partage des tâches familiales) ou d'ordre moral (encouragement).}

\section{Environnement personnel : lien travail-famille}

Plusieurs commentaires intéressants ont été formulés au cours des entretiens au sujet de la conciliation entre le travail et la famille. Par exemple, les répondants s'entendaient pour dire que le soutien de leur conjoint avait été d'une grande importance dans le succès de leur entreprise et de leur famille. L'appui apporté par le conjoint pouvait être d'ordre physique (implication directe dans le commerce ou partage des tâches familiales) ou d'ordre moral (encouragement). Certains répondants ont aussi souligné l'importance de leur famille (outre le conjoint) dans leurs efforts pour concilier leurs responsabilités à l'égard de leur famille et de leur entreprise. Les passages suivants, tirés des entretiens, reflètent ces observations :

J'ai une femme très ouverte et compréhensive. Elle $s$ 'adapte vite et est très flexible. Elle comprend que je 
doive parfois travailler tard. Elle $m$ 'aide également dans le commerce; c'est elle qui s'occupe de la comptabilité du magasin. (A.L.)

Ça prend beaucoup d'organisation et de planification. Mais au quotidien, maintenant que j'ai une famille - j'ai trois enfants -, c'est vraiment difficile. J'ai dî m'ajuster. Ça prend un conjoint qui collabore. On partage beaucoup les tâches familiales. Mon mari travaille plutôt les fins de semaine et le soir, ça fait qu'on roule tout le temps! On se fait des horaires et une liste des choses à faire. (C.C.)

Nous autres, on est tous impliqués. On est trois : moi, mon mari et ma fille. Ma fille travaille à temps plein et mon mari m'aide quand c'est possible. Je crois que lorsqu'une personne commence en affaires, il lui faut avoir l'appui de son conjoint. Si, au départ le conjoint est réticent vis-à-vis de l'entreprise que tu envisages de démarrer, ce n'est pas bon. Le conjoint doit être d'accord et donner son appui, peu importe si c'est par du travail physique ou juste du soutien moral. (M.R.)

Ça prend du soutien familial parce que, souvent, il arrive qu'on travaille tard. Il faut partager les tâches avec sa femme et faire un peu sa part. (G.B.)

$C$ 'est très important d'impliquer la famille et d'avoir son appui. Sinon, c'est impossible d'avoir une entreprise comme la mienne, surtout dans le genre de travail que je fais. (H.L.)

Quant aux quatre répondantes qui étaient chefs de familles monoparentales, elles ont dû apporter certains ajustements à leur situation de manière à pouvoir concilier leur vie familiale et professionnelle. Leur comportement démontre bien que ce sont les femmes qui doivent veiller au bien-être des enfants advenant le départ du père. L'équilibre familial constitue donc un élément important pour les femmes, même lorsqu'elles se trouvent déjà en affaires. Cette dernière constatation dénote l'importance de l'équilibre familial pour les femmes d'affaires. Par exemple, après leur divorce, deux de ces répondantes ont installé leur bureau dans leur maison de manière à être plus près de leurs enfants. Voici comment une de ces personnes a raconté son expérience :

Je vais vous dire: ça, c'est un aspect sur lequel je travaille toujours. Ce n'est pas aussi évident que ça en a l'air. Après ma séparation, j'ai installé mon bureau au sous-sol de ma maison pour être plus proche de mes enfants. Lorsque les enfants arrivaient de l'école, je montais en haut leur faire faire leurs de- voirs et je faisais le souper, puis je redescendais travailler. Je vais vous dire que j'ai travaillé ! Et puis, il y a encore du travail à faire. (J.L.)

L'une de ces répondantes était propriétaire d'un restaurant. À cause de la nature même de son commerce, il lui était impossible de l'installer dans son sous-sol. Ce sont donc ses enfants qui sont allés la trouver dans son commerce. Voici ce qu'a raconté cette répondante :

Il faut avoir un équilibre [...] Comme mère de famille, quand j'ai élevé mes enfants, ils pouvaient venir me voir au restaurant quand ils le voulaient. Ils venaient me trouver au restaurant après l'école et faisaient leurs devoirs dans la cuisine. C'était leur maison. Du mois d'octobre au mois d'avril, je faisais toujours les soupers de famille au restaurant [...] Même si je faisais de longues heures, les enfants pouvaient venir ici en tout temps. On avait notre vie familiale à l'intérieur du commerce, et le samedi avant-midi était consacré au ménage de la maison. (R.T.)

Quant à la troisième répondante de ce groupe, c'est à sa grande énergie qu'elle attribue le succès avec lequel elle a concilié ses diverses responsabilités :

J'ai toujours fait cela. Lorsque j'avais une jeune famille, je réussissais bien à concilier tout ça. Je mettais beaucoup d'heures de travail. Ce n'est pas pour vous insulter, mais je pense que les femmes ont plus d'énergie que les hommes. Nous sommes capables de faire deux jobs à la fois. Je pouvais sortir de mon commerce, aller chercher les enfants, les faire manger, les emmener au hockey, faire leurs devoirs avec eux, aller aux réunions parents-maîtres. En plus, j'étais dans différents comités. C'est dans ma nature de faire cela. Je suis habituée à faire plusieurs choses à la fois. C'est ça l'avantage : j'ai beaucoup d'énergie. Mon conjoint n'a pas pu m'aider, car il n'était pas là. J'ai tout fait seule. Maintenant, j'ai un nouveau conjoint dans ma vie, mes enfants sont grands et sont partis. Je pense que mes enfants sont plus débrouillards cependant. (H.H.)

Un seul homme entrepreneur avait vécu une séparation, et c'est son ex-épouse qui a obtenu la garde des enfants. Ce répondant a avoué que le temps consacré à son commerce au début avait eu raison de son mariage. Cependant, cette séparation n'empêchait pas les enfants de travailler pour lui pendant leurs vacances d'été. 


\section{L'implication des enfants dans le fonctionnement du commerce est une autre caractéristique qui est ressortie des entretiens.}

Justement, l'implication des enfants dans le fonctionnement du commerce est une autre caractéristique qui est ressortie des entretiens. Dans la mesure du possible, les répondants ayant des enfants en âge de travailler tentaient de les faire participer à l'entreprise moyennant une rémunération. Curieusement, dans tous les cas répertoriés sauf un, il s'agit de femmes. C'est à se demander s'il n'existe pas un lien entre l'implication des enfants dans le fonctionnement du commerce sous la supervision de leur mère et le fait qu'un nombre important de répondants aient mentionné, comme nous l'avons vu plus tôt, que c'était principalement leur mère qui leur avait inculqué les valeurs liées au travail et certains traits de caractère. Les femmes seraient-elles plus patientes que les hommes ? Ou encore, considèrent-elles cette tâche comme le prolongement de leur rôle de mère ? Voici des extraits d'entretiens à ce sujet :

J'ai un garçon de 23 ans qui travaille chez nous. Je l'ai impliqué dans la business assez jeune. Je lui ai inculqué des notions assez tôt, mais je ne sais pas s'il va continuer dans la même direction. Il est tout à fait le contraire de moi, il n'est pas organisé. Le bureau principal est dans ma maison et mon fils fait plusieurs tâches pour la business et je le paye pour ses services comme un employé. (R.V.)

Mes jeunes ont commencé très jeunes à travailler ici, au restaurant, jusqu'à l'âge d'aller aux danses. Je leur donnais 10 \$ pour aller à la danse, et pour me rembourser, ils devaient venir laver la vaisselle après que la danse était finie. C'est de même que ça marchait. (R.T.)

J'ai toujours impliqué les enfants dans de petites tâches et des plus grandes lorsqu'ils ont grandi, et je les ai payés. Ça n'a donc pas été un problème d'avoir de l'aide d'eux. Et j'ai toujours exigé $100 \%$; je voulais que les étiquettes soient droites, et il fallait qu'elles soient bien droites. Ils ont appris eux aussi à livrer le produit très jeunes. (H.L.)

Enfin, la majorité des répondants ont déclaré ne pas avoir le temps de faire d'autres activités. Certains ré- pondants ont même avoué qu'ils n'avaient pas de vie sociale et que leur vie familiale, sociale et professionnelle tournait autour du commerce. Voici des extraits de leurs commentaires à ce sujet :

Quand j'ai accepté ce poste, ça été un soulagement, car l'entreprise fonctionne très bien sans que je sois là, elle réussit très bien. Je n'ai pas besoin d'être là. L'entreprise faisait partie de ma vie sociale, personnelle, familiale et communautaire. Ça fait que la famille faisait partie de cela, les enfants ont participé et ont toujours vu ce que je faisais. Je trouvais important que la famille soit ensemble, surtout qu'elle avait passé à travers un divorce. On ne peut pas appeler ça un équilibre, car j'ai travaillé comme une damnée. La famille était toujours là, par exemple, même qu'à un moment donné la famille faisait partie de l'entreprise. Il n'y avait que le travail et la famille, je n'avais pas de vie sociale. Mes amis faisaient partie de mon équipe. (J.L.)

On travaille tellement dans nos commerces qu'on se fait une vie sociale à partir du commerce. C'est tout mélangé ensemble. Ma vie sociale, elle est avec mes clients. (R.T.)

Toute la famille participe à l'entreprise. Les enfants travaillent avec nous l'été et ils peuvent ainsi gagner un peu d'argent, car les jobs sont tellement rares dans la région. Moi, je me lève à 5 heures le matin et je travaille jusqu'à 10 heures le soir. Ma femme, c'est la même chose. Notre vie familiale tourne autour de notre commerce. (A.S.)

\section{Conclusion}

De façon générale, les renseignements recueillis dans cette étude qualitative ont été très utiles pour mieux comprendre les caractéristiques personnelles des entrepreneurs ainsi que l'influence de leur environnement personnel sur leur comportement.

Par exemple, parmi les facteurs qui ont prédisposé les répondants vers une carrière entrepreneuriale, la présence de parents en affaires est un élément important puisque 18 des 28 répondants ont affirmé avoir un ou des parents en affaires. L'existence de parents (surtout d'une mère) dynamiques (six répondants) et l'acquisition d'une solide expérience tout au long de leur vie (quatre répondants) ont été les autres facteurs exprimés par les répondants pour tenter de justifier leur présence en affaires. 
Quant à l'environnement personnel l'analyse des résultats fait ressortir les trois observations suivantes : premièrement, le conjoint ainsi que la famille de l'entrepreneur jouent un rôle très important dans le succès de l'entreprise; deuxièmement, les enfants sont encouragés jeunes, par leur mère surtout, à travailler dans l'entreprise familiale; troisièmement, les efforts consacrés par les répondants à leurs responsabilités familiales et d'affaires occupent tout leur temps. De plus, les répondantes qui sont parent unique ont dû apporter des ajustements à leur environnement de manière à être plus près de leurs enfants.

\section{Parmi les facteurs qui ont prédisposé les répondants vers une carrière entrepreneuriale, la présence de parents en affaires est un élément important}

Les résultats sur l'environnement personnel de l'entrepreneur sont conformes aux préoccupations relevées dans la littérature au sujet de l'importance du soutien familial dans le succès de l'entreprise et à l'effet que ce sont les femmes qui doivent le plus souvent s'adapter aux changements occasionnés par les responsabilités additionnelles liées à l'entreprise.

\section{Notes et références}

1 Yves Robichaud est professeur à l'École de l'administration et du commerce de l'Université Laurentienne à Sudbury, Ontario. Egbert McGraw est professeur à la faculté d'administration de l'Université de Moncton, au Nouveau-Brunswick.

2 Voir à ce sujet les modèles développés par : Blawatt, K. «Defining the Entrepreneur : A Conceptual Model of Entrepreneurship » (1995). Rapport du congrès annuel du Conseil canadien de la petite et moyenne entreprise, p. 1337; Naffziger, D.W., J.S. Hornsby et D.F. Kuratko (1994). «A Proposed Research Model of Entrepreneurial Motivation ", Entrepreneurship Theory and Practice, vol. 18, $\mathrm{n}^{\circ} 3$, p. 29-41; Herron, L. et R.B. Robinson (1993). «A Structural Model of the Effects of Entrepreneurial Characteristics on Venture Performance ", Journal of Business Venturing, vol. 8, $\mathrm{n}^{\circ}$ 3, p. 281-294; Keats, B.W. et J.S. Bracker (1988). « Toward a Theory of Small Firm Performance : A Concepttual Model», American Journal of Small Business, printemps, vol. 12, $\mathrm{n}^{\circ} 4$, p. 41-58; Hollenbeck, J. et E. Whitener (1988). « Reclaiming Personality Traits for Personal Selection », Journal of Management, vol. $14, \mathrm{n}^{\circ} 1$, p. $81-91$.
3 Les caractéristiques personnelles représentent dans notre étude les facteurs historiques susceptibles d'influencer la présence des individus en affaires, tandis que l'environnement personnel correspond au lien travail-famille.

4 Blawatt, K. (1995), op. cit., p. 13-37.

5 Greenberger, D.B. et D.L. Sexton (1988). « An Interactive Model of New Venture Initiative », Journal of Small Business Management, vol. 26, $\mathrm{n}^{\circ}$ 3, p. 1-7.

6 Shapiro, A. (1984). The Entrepreneurial Event, The Environment for Entrepreneurship, Calvin, A. Kent, Ed., Lexington Books, D.C. Heath and Company, Lexington, Mass. Toronto, p. 21-40.

7 Liang, C.K. et P. Dunn (2003). «Love, Live, and Family ties : Couples' Assessment of New Venture Creation and Business Development and Family Relationships », Association for Small Business \& Entrepreneurship Proceedings; Cachon, J.C. (1992). «Entrepreneurs : pourquoi ? Comment? Quoi ?», Revue du Nouvel-Ontario, vol. 13, p. 13-56.

8 Blawatt, K (1995), op. cit.

9 Das, M. (2001). « Women Entrepreneurs from India : Problems, Motivations and Success Factors ", Journal of Small Business and Entrepreneurship, vol. 15, n 4, p. 6781; Ferguson, F.E. et J.R. Durup (1997). « Work-family Conflict and Entrepreneurial Women: A Litterature Review », Journal of Small Business and Entrepreneurship, vol. 14. $\mathrm{n}^{\circ}$ 1, p. 30-51; Newcomer, J. et S. Taylor (2003). "Why Superwoman is Starting her Own Business Today », Association for Small Business \& Entrepreneurship, Proceedings; Lee-Gosselin, H. et J. Grise (1990). « Are Women Owner-managers Challenging our Definitions of Entrepreneurship ? An In-depth Survey », Journal of Business Ethics, vol. 9, $\mathrm{n}^{\text {os }} 4$-5, p. 423-435; Neider, L. (1987). "A Preliminary Investigation of Female Entrepreneurs in Florida », Journal of Business Management, vol. 25, $\mathrm{n}^{\circ} 3$, p. 22-29.

${ }^{10}$ Newcomer, J. et S. Taylor (2003), op. cit.

11 Carter, N. (1997). « Entrepreneurial Processes and Outcomes. The Influence of Gender », in P.D. Reynolds et S.B. White, Quorum, The entrepreneurial Process : Economic Growth, Men, Women, and Minorities.

12 Belcourt, M., R. Burke et H. Lee-Gosselin (1991). The Glass Box: Women Business Owners in Canada, Ottawa, The Canadian Advisory Council on the Status of Women. Neider, L. (1987), op. cit., p. 22-29 ; Bullers, S. (1999). « Selection Effects in the Relationship between Women Work-family Status and Perceived Control » Family Relations, vol. 48, $\mathrm{n}^{\circ}$ 2, p. 181-188. Scarborough, N.M. et T.W. Zimmerer 
(2000). Effective Small Business Management, $6^{\text {th }}$ Edition, New Jersey, Prentice-Hall, Inc.

13 Liang, C. K. et P. Dunn (2002). « The Impact of Starting a New Venture on the Entrepreneurs and their Families: Expectations, Reality, and Willingness to Start again », Journal of Business and Entrepreneurship, vol. 14, $\mathrm{n}^{\circ}$ 1, p. 34-46; Scarborough, N.M. et T.W. Zimmerer (2000), op. cit.; Ferguson, F.E. et J.R. Durup (1997), op. cit., p. 30-51.
14 Danes, S., V. Zuiker, R. Kean, J. Arbuthnot et K. Kaye (1999). « Predictors of Family Business Tensions and Goal Achievement» Family Business Review, vol. 12, n 3, p. 241-251; Davies, J. (1999). « Mother of Invention : Women Entrepreneurs », ManagementToday, January, p. 6466; Page, H. (1999). « Entrepreneur with Children : Stringing a Balance between Business and Family», Entrepreneur, vol. 27, $\mathrm{n}^{\circ} 4$, p. 14-15. 


\section{Publicité}

ROT - Vous désirez recevoir un de nos numéros déjà parus ? 